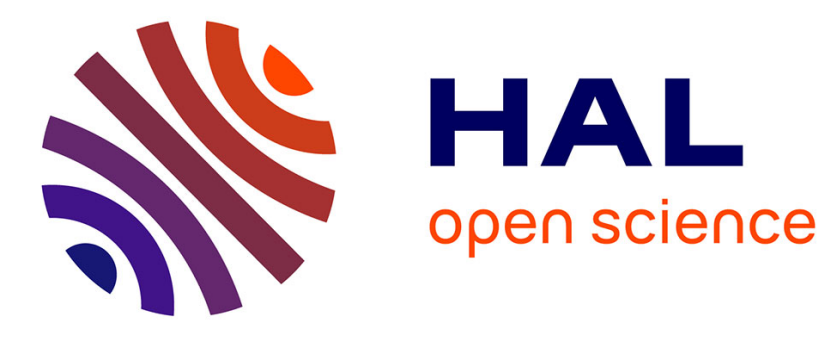

\title{
Agent-Based Coalition Formation in a Co-modal Transport System
}

Zhanjun Wang, Khaled Mesghouni, Slim Hammadi

\section{To cite this version:}

Zhanjun Wang, Khaled Mesghouni, Slim Hammadi. Agent-Based Coalition Formation in a Co-modal Transport System. 2014 IEEE/WIC/ACM International Joint Conferences on Web Intelligence (WI) and Intelligent Agent Technologies (IAT), Aug 2014, Warsaw, Poland. 10.1109/WI-IAT.2014.182 . hal-01716163

\section{HAL Id: hal-01716163 \\ https://hal.science/hal-01716163}

Submitted on 27 Feb 2018

HAL is a multi-disciplinary open access archive for the deposit and dissemination of scientific research documents, whether they are published or not. The documents may come from teaching and research institutions in France or abroad, or from public or private research centers.
L'archive ouverte pluridisciplinaire HAL, est destinée au dépôt et à la diffusion de documents scientifiques de niveau recherche, publiés ou non, émanant des établissements d'enseignement et de recherche français ou étrangers, des laboratoires publics ou privés. 


\section{Agent-based Coalition Formation in a Co-modal Transport System}

\author{
Zhanjun Wang \\ LAGIS, Ecole Centrale de Lille \\ Cité Scientifique CS 20048 \\ 59651 Villeneuve d'Ascq, France \\ Email: zhanjun.wang@ec-lille.fr
}

\author{
Khaled Mesghouni \\ LAGIS, Ecole Centrale de Lille \\ Cité Scientifique CS 20048 \\ 59651 Villeneuve d'Ascq, France \\ Email: khaled.mesghouni@ec-lille.fr
}

\author{
Slim Hammadi \\ LAGIS, Ecole Centrale de Lille \\ Cité Scientifique CS 20048 \\ 59651 Villeneuve d'Ascq, France \\ Email: slim.hammadi@ec-lille.fr
}

\begin{abstract}
This paper is aimed at presenting a service formation process in a transport information system which is dedicated to the co-modal transportation service. The service formation is achieved via the coalition formation to get a full itinerary with identified transportation service. Meanwhile, the coalition formation targets to achieve the optimum combination with respect to the user's preferences. The multi-section(transfer) characteristics of the journey and numerous simultaneous requests make it necessary to apply a multi-agent approach. With the role-switching of the agents, the agent behaviors and protocol are established to normalize each step of the agent coalition.
\end{abstract}

Keywords-co-modal transport; service formation; multi-agent system; coalition protocol

\section{INTRODUCTION}

In a multi-agent system, when the agents have to carry out tasks that they can't perform individually, the coalition formation is necessary. Thus, the coalitions can be employed as a means for multi-agent cooperation and collaboration. Usually, the coalition formation allows the agents to perform tasks that are beyond their own ability.

To establish this union of agents, several coalition formation models have been proposed [1] [2] [3] [4] [5]. Based on different assumptions, these coalition formation models are proposed in different problem context. The agents are divided into self-interested agents [6] and group-oriented agents [5]. The first type of agents try to get their individual utilities maximized while the group-oriented agents aim at optimizing group utility. In this work, with the special problem characteristics, we will develop a coalition formation mechanism with the self-interested agents to maximize the global utility.

In our problem, the aim of the coalition formation is to form a route of several solutions following a user's request. Obviously the itinerary should be composed of more than one sections (otherwise there is no need of coalition). Each member section is represented by one agent.

The coalition formation process begins with an initiator. In our approach, the possible initiators of the coalition are known since the assignment for the itineraries are identified [7]. The agent that represents the first section of the itineraries will play the role of initiator if the coalition formation process conducts in the forward direction, otherwise the agent of the last section which contains the destination point will play as the initiator.
The article is organized as follows. Section II contains the context of the problem and the description of the problem. In Section III, our approach for the coalition formation is given in details where the protocol of the interaction is described. In section IV, an example is given to illustrate the method. In the last Section, conclusions and future works will be presented.

\section{THE PROBLEM}

In this section, we will describe the context of the problem and define formally this coalition formation problem.

\section{A. Context of the problem}

In this part, a three layers model of the proposed solution for the co-modal transportation problem will be presented. From the users' itineraries requests, the global optimal itineraries will be obtained [8] [9]. Each itinerary composes of one or more segments that are not necessarily ensured by the same transport operator, and there are several possibilities to choose the transportation tools for each segment. The itineraries may also have segments in common. In this case, the travelers share the same segment of the entire route. In particular, the resource (e.g., the carpooling and the freeuse cars) is limited. To get better allocation of the available resource and to well arrange the travelers' journey according to their preferences, a three layers model is implemented. Figure 1 presents a diagram illustrating the relation of these layers and the relevant activities.

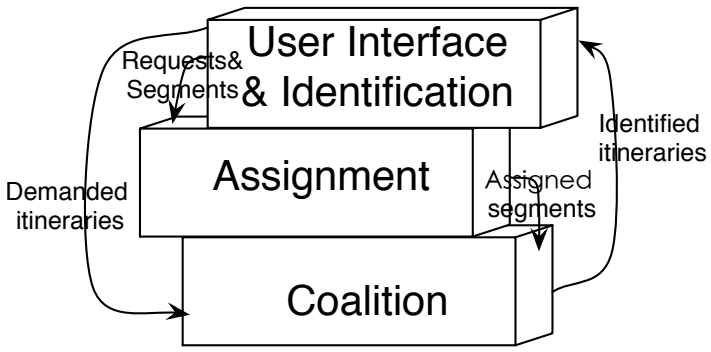

Fig. 1. The layered structure of the model

The first layer is served as the interface and the identification of global itineraries. The identified itineraries will be decomposed according to the areas, the transport operators and the service modalities (here the term "the service modalities" means the mode of the transport service, like public 
transport, carpooling). Thus, the decomposed segments and the demanded itineraries will be sent to the second layer and the third layer. In the second layer, the assignment for each traveller will be accomplished in the limit of available source of transportation service and the travelers' preference in an optimal way. In the second layer, the modality will be chosen in an appropriate way for each traveller in each segment. Once this step is done, the allocation is finished. In addition, each segment of path is represented by an agent of the multi-agent model in this level. In the third layer, the assigned segments and the demanded itineraries permit the coalition among the routes to formulate the entire journey which will be displayed on the users' terminal. The third layer is the last step to form a whole itinerary from the allocation gotten in the second layer. This step will be on the basis of some interaction protocol. The process of getting the segments allied is named in this paper combination of the segments. The agents of the multiagent system cooperate from the first step of the receiving of the requests to the last step of sending back the results to the transportation demanders. Several types of agents are created for the different missions. All these will happen in a context of Multi-Agent System. By the way, each layer contains a process of optimization.

Figure 2 is the entire activity diagram of the transport information system. The last part which is marked grey is the work of this paper. With the work on the previous steps, this paper focuses on the coalition combination of the identified route sections.

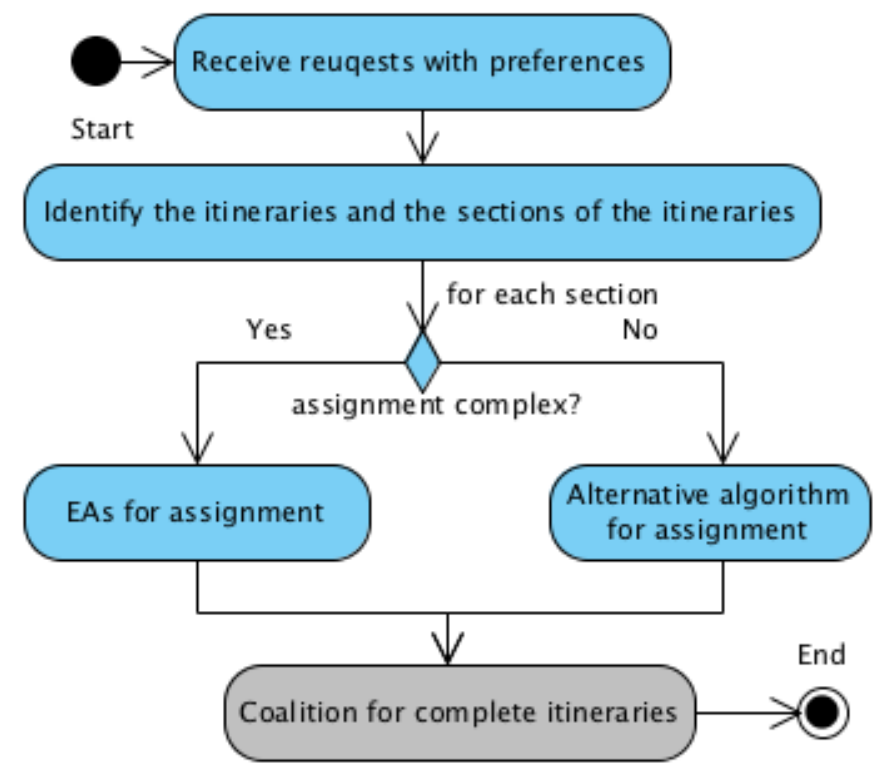

Fig. 2. The entire flow chart of the information system

In the previous work [7], we have described the assignment details for itinerary request. The results can be used in this work is the identified itineraries with the possible assignment for each section of it. The potential transportation service to ensure each segment of the itinerary is already known. The purpose of the coalition is to find out the optimal solution for each journey, and it means that with respect to the preference and the availability, the coalition process permits an optimal solution according to the criteria.
In the coalition practice, each section of the route is represented by an agent and the problem becomes the coalition of agents. Since the conditions may be contradictory and the best coalition (maybe several) is unpredictable, in this work, it's addressed through multi-agent coordination. We try to set up a protocol for the coalition process. For example, one agent will send the specified information to certain agents to avoid useless communication and in the presence of contradictory conditions, the agent should be able to make a compromise following the criteria to formulate a valid solution. In [13], the compromise strategy is applied for selecting coalitions, accepting proposals and distributing the revenue during the coalition formation process. Then we introduce a model which provides the agents with a dynamic coordination mechanism. This will define the steps to follow for a complete coalition formation. Given an itinerary and the relevant assignment for its sections, the agent that serves as sponsor will initiate the formation and the structure for the coalition is also ready to use.

Each section of route which has been well assigned in the last step of work will be represented by an agent. Later in this paper, it is named as Route Agent.

\section{B. Problem description}

Consider a set of agents $A=\left\{a_{1}, a_{2}, \ldots, a_{n}\right\}$ and a set of tasks $K=\left\{k_{1}, k_{2}, \ldots, k_{m}\right\}$, the agents in $A$ can execute the tasks in $K$ and negotiate over their coalition to execute a combination of tasks in $K$ [10].

Each agent $a_{i}$ possesses a set of resources $s_{a_{i}}=$ $\left\{s_{a_{i}}^{1}, \ldots, s_{a_{i}}^{p}\right\}$ which is consisted of $p$ different types. These $p$ types of resources have the same capacity to perform a task but with different result. If $a_{i}$ is able to execute a task $k_{j}$, one resource $s_{k_{j}}$ of $s_{a_{i}}^{l}(1 \leq l \leq p)$ is required. To perform a bundle of tasks which are similar with $a_{i}$ at the same time, the same agent $a_{i}$ is needed. The resource possessed by an agent is always limited, the pre-allocation of the resource is ready to be used in our work. Some tasks form an union and can't be divided. These tasks make up a completed whole task. Besides, these tasks in the union should be performed strictly in a certain order. To execute this union of tasks, an agent don't always be able to perform it. A chain of agents will combine and put their resources in common in order to perform this union of tasks together.

The set of resources required to perform the group $g$ of $m$ tasks is the sum of resources required for each task in $g$, i.e., $S_{g}=\left\{s_{g_{1}}, \ldots, s_{g_{m}}\right\}$ with $s_{g_{l}}$ the resource needed for task $g_{l}(\forall l, 1 \leq l \leq m)$.

The agent $a_{i}$ is related to the route $R$ and we can interpret this in the following way: the route $R$ is a task $k$ which can be executed by the agent $a_{i}$. The coalition formation for the itineraries becomes the coalition of the agents to execute the set of tasks. The goal of an agent is to find out the coalitions to which it belongs, such that the global utility is maximized. At the end, a set of agents will be combined exactly for the execution of a set of tasks, meanwhile, these tasks form an entire itinerary.

In a global view of the coalition formation, for all the requests at the same time, the tasks form a set and the agents 
form a set. There is also a pre-allocation of the resources for the tasks. The coalition formation is used to form itineraries for all the requests. As one agent possesses the resources, it can be used repeatedly to perform the task in the limit of its available resources.

In a detailed view, for one request, the agents try to form a set and combine together to execute a group of tasks with their resources for a complete itinerary. After that the coalition formation is completed and taken, the coalition consumes the resources and the related agents which have participated in this process update the remain resources.

\section{Definitions}

We introduce some definitions before presenting the details about the behaviors and coalition formation protocol.

Definition: Route agent. An agent that is related a route section with its detailed information.

The Route Agent carries the following information: the travelers on this section, the route information(the origin, the destination, the distance etc.), the vehicles on this route, the assignment result in our work.

Definition: Coalition. A set of agents that combine among themselves to execute the tasks.

Definition: Coalition formation. The process to establish an agent coalition.

Definition: Coalition structure. A task structure that concludes the tasks to be executed and their relationships. According to it, route agents interact for a coalition formation.

In this problem, the coalition structure is the decomposed itinerary of the request, along with the user's specific preferences. In fact, there are two ways to develop the coalition: from origin to destination and from destination to origin. Generally speaking, the first method is often applied. But when the condition about the latest arrival time is imposed, the second method that starts the coalition from the destination to the origin is chosen.

Definition: Coalition formation protocol. The protocol which has to be followed by the agents during the coalition formation.

The protocol defines the main steps (phases) of the coalition formation.

Definition: Behavior strategy. In each step of the coalition formation, the behavior strategy describe how the agents perform.

In the process of coalition formation, not only the agents that can execute the tasks are indispensable, but also the agents for the coordination and evaluation of the coalition are necessary.

We develop a type of agent: evaluation agent. This agent is used to evaluate one coalition that represents an itinerary for the traveller. After that one coalition formation process ends for a complete itinerary, the evaluation agent will take charge of the coalition to calculate the relevant indicators for the itinerary: travel time, total expense, waiting time, number of transfers etc. This agent allows to compare the different results, thus choosing one.

Definition: Utility function. A function that measures the coalition in terms of some defined criteria like cost and time.

To evaluate the quality of the coalition for the demand, the utility function is used to measure the result of the coalition formation. The result is about the resource that the coalition takes. The coalition evaluation agent works with it. The utility function may vary for each coalition because of the different criteria imposed on the requests. This function may take the conditions like the total expense, the travel time, the number of transfers, the waiting time as parameters, or an aggregation of several ones.

There are several roles that an agent plays in participating in an entire coalition formation.

Definition: Candidate: The role of an agent when it doesn't belong to any coalition. Before an agent engages in a coalition, every agent is a candidate for any coalition.

Definition: Ready_up: The role of an agent when it is selected for one coalition.

Definition: Member: The role of an agent when it has joined one coalition.

Definition: Leader: The role of an agent when it initiates an coalition.

Definition: Terminator: The role of an agent when it performs as the last one of a coalition.

During the coalition process, the role of an agent may switch from one to another, i.e., an agent candidate may become leader if it has the profile to launch a coalition or an agent member may become candidate again if it leaves an existed coalition.

\section{COALITION FORMATION APPROACH}

According to the conditions imposed by the service demanders, there may be several itineraries for one journey. For example, the shortest itinerary may be different from the one with the least expense. These itineraries are the coalition structures with which the formation of the service follows.

The obtained assignment result is in form of matrix, as Table I. Each row of the matrix refers to the transportation service demander and each column of the matrix refers to the description of the transportation type. As the matrix shows, it's about several travelers in the same assignment result. Thus, the agent representing this result can take part in all the involved travelers' coalition formation of the itenerary. For example, according to the assignment result in Table $\mathrm{I}$, the service demander $P_{3}$ will take the vehicle $V_{2}$ to travel from $A$ to $B$ during $\left[t_{A_{2}}, t_{B_{2}}\right]$ with the expense of $C_{2}$.

TABLE I. MATRIX OF AN ASSIGNMENT

\begin{tabular}{|c|c|c|c|}
\hline $\mathrm{R}(\mathrm{A}, \mathrm{B})$ & $V_{1}\left(t_{A 1}, t_{B 1}, C_{1}\right)$ & $V_{2}\left(t_{A 2}, t_{B 2}, C_{2}\right)$ & $V_{3}\left(t_{A 3}, t_{B 3}, C_{3}\right)$ \\
\hline$P_{1}$ & 0 & $*$ & 1 \\
\hline$P_{2}$ & 1 & $*$ & 0 \\
\hline$P_{3}$ & $*$ & $\boldsymbol{I}$ & $*$ \\
\hline$P_{4}$ & 0 & $*$ & 1 \\
\hline
\end{tabular}


For a given request, when the assignment of the vehicles has been completed for each section of the route, the coalition formation process can be launched. The global algorithm for this process is shown as Algorithm 1.

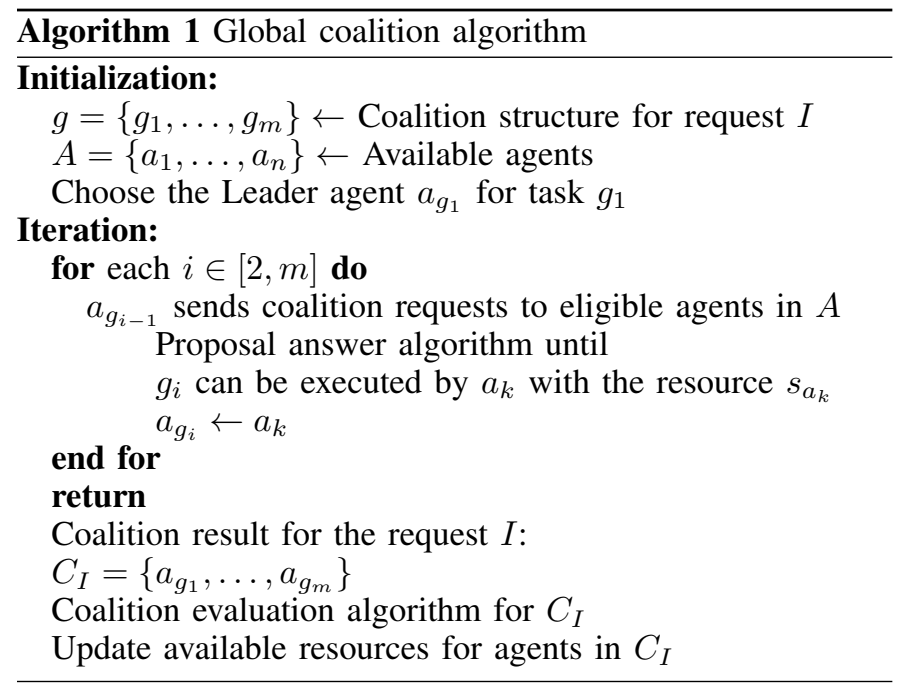

\section{A. Coalition formation model}

For the start-up of the coalition formation, there are two options: to begin with the origin of the journey and to begin with the destination of the journey. In a general way, if there is one condition on the latest arrival time at the destination, the coalition formation process begins with the last section of the itinerary. Otherwise, the coalition formation picks the section with the origin of the itinerary as the first part of the coalition.

\section{B. The agent behaviors}

During the coalition formation process, the negotiation between the agents is performed on their own, which means, there is no external decision maker or central algorithm. With respect to the preference and criteria, the agent decides locally its action. Some behavior strategies will be proposed in the following and allow the negotiation of the agents.

The coalition formation commences when an agent is chosen and publishes a request. This agent plays the role of a leader. All the available candidate agents that well match the published request will adopt the ready_up role [11]. For each ready_up agent, it will reactively respond to the requests.

Each agent sends different type of proposals to other agents and also receives relevant responses. The agents need behavior strategies:

- $\quad$ Sending proposals.

- $\quad$ Receiving message.

- $\quad$ Responding to the message.

This activity refers to coalition request conversation as described in Figure 3.

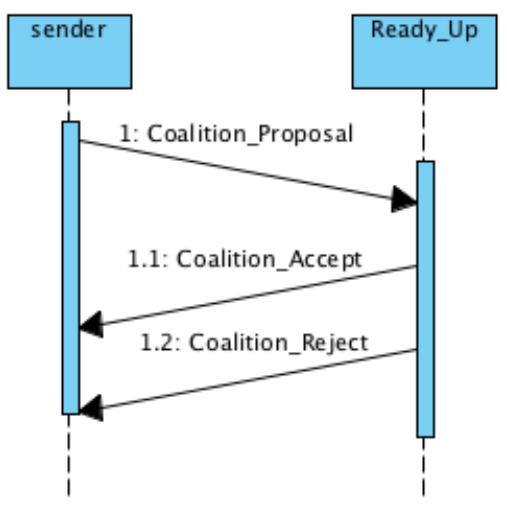

Fig. 3. Coalition proposal conversation

The condition on nodes totally depends on the coalition structure which has the full itineraries for the journey and the connections or transfers have already been determined.

For the route section $S_{m}$, it represents the route $\left[S_{m O}, S_{m D}\right]$, where $S_{m O}, S_{m D}$ are respectively the origin and the destination. According to the coalition structure, the next section is $S_{m+1}$,

Apart from the constraints imposed by the service demanders, the important condition about time for the coalition is the order: $t_{\text {LastArrival }}<t_{\text {Departure }}$.

The sender transmits a coalition proposal to the eligible agents which meet the precedent conditions on time and on transfers. These eligible agents are also named ready_up agents.

If an agent ready_up accepts the coalition proposal, it will send back a coalition acceptance message to the sender. The ready_up agent becomes member agent of the coalition. Otherwise, it sends back a coalition rejection message as feedback. The agent returns to its candidate agent status.

To be able to become a member agent of the coalition, the following conditions should be satisfied:

- The nodes of the route coincide with the due nodes of coalition structure.

- The traveller is well attributed a vehicle on this section of route in the assignment matrix.

- The arrival time of the vehicle is not after the departure time on the following route.

After the confirmation of the member status, the resource of the agent updates.

The activity of finding the next member of the coalition refers to adding new member to the coalition, as described in Figure 4.

Without loss of generality, the coalition is started from the origin to the destination of the itinerary.

As to how to identify the leader of the coalition, we have to refer to the first section (respectively, the last section for the coalition from destination to origin) of the coalition structure. Apart from the conditions on the nodes, the requirement of 
time should also be met (if there is any imposed earliest departure time).

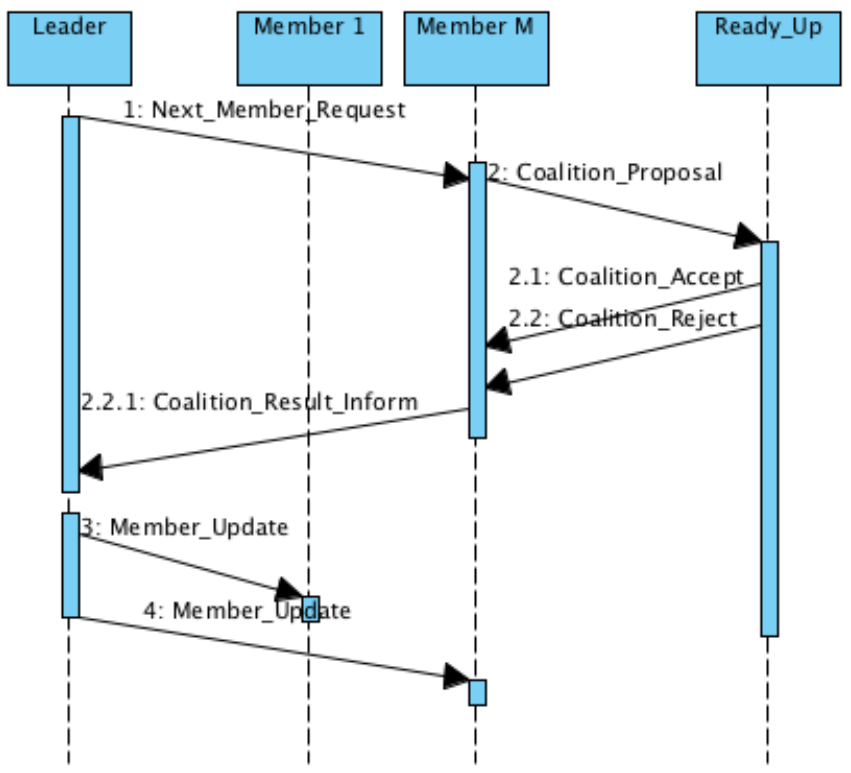

Fig. 4. Add new member to the coalition

Leader agent sends the request of next member to the following member of the coalition, with reference to the coalition structure. The receiver of the request sends the coalition proposal to the qualified candidates. The ready_up agent will respond proactively. In this step, the coalition proposal conversation will be repeated until a member is found. After the response from the ready_up to the member agent's proposal, a coalition result inform message is sent to the leader agent of the coalition. Then, all member agents are informed of the update information by receiving member update message.

The activity of verifying the termination of the coalition refers to terminate the coalition formation as described in Figure 5.

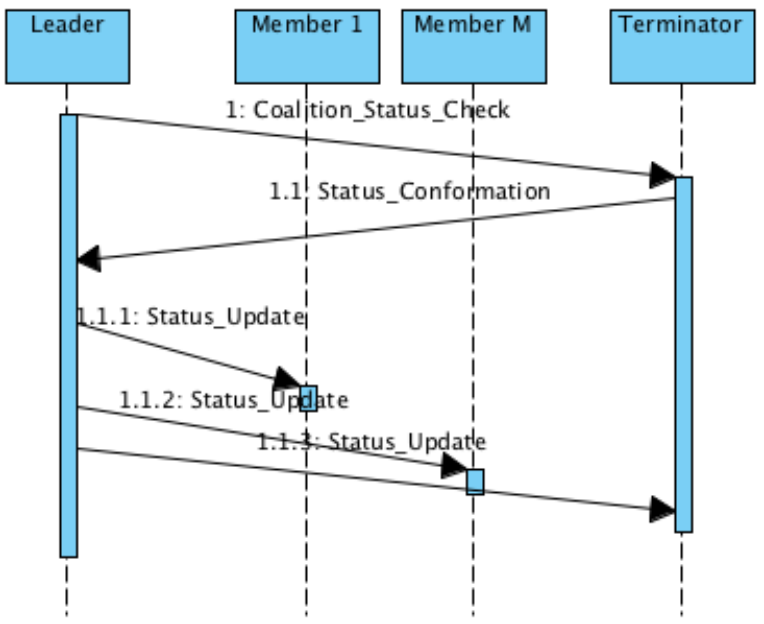

Fig. 5. Terminate the coalition formation

Leader of the coalition sends coalition status verification request to the last agent. After having checked the conditions, it replies to the leader with coalition status confirmation message. If the last agent of the coalition confirms the end of the coalition formation process, this member agent becomes terminator agent.

To choose the terminator of the coalition formation, the following conditions should be satisfied:

- The end point of the route represented by terminator agent is the same with the destination of coalition structure.

- The condition on the arrival time: $t_{\text {arrival }}<$ $t_{\text {arrivalBefore }}$, where $t_{\text {arrival }}$ denotes the real arrival time and $t_{\text {arrivalBefore }}$ denotes the imposed latest arrival time.

After that the terminator agent is chosen and the coalition is completed. The evaluation agent will send the results.

During the entire coalition formation process, the evaluation agent works at each step of the combination. The preferences of the demander may vary from one to another. There may exist several possibilities for the final eligible results, thus the optimization becomes necessary to find the most suitable one.

The criteria consists of the travel time, the total expense, the number of transfers and the waiting time.

Now that the service demanders emphasize different criteria, for example, the lest expense, the least travel time, the least transfer number or even an aggregation of several conditions. The local evaluation will be executed.

The coalition formation process ends when one of the following conditions is met:

- The coalition has a terminator agent.

- The leader agent abandons the coalition.

- $\quad$ The predefined deadline is due.

Once a coalition formation ends and the related solution is chosen by the demander, the general situation such as the availability (the available number) of the vehicles updates.

\section{Compromise}

In some case, the imposed criteria are so strict that they prevent the agents from forming a valid coalition. For example, during the given interval of travel time, there isn't any suitable solution. Like in humans society, people choose sometimes to compromise their profit if a solution is agreeable to all participants and may bring them benefit. In the agents world, to provide the service demander with a practicable advice, certain compromise to some extent should be made to achieve one solution.

Frequently, the reasons that lead to the no-solution are among the followings:

- The criteria on the mode of transport (type of service) are too strict and there isn't enough resource for the allocation.

- The condition on the travel time is too limited.

- There is not any suitable solution. 


\section{Coalition formation protocol}

The coalition formation protocol, consisting of an evaluation agent and multiple route agents, defines the procedures to follow, from the start-up to the termination of the coalition formation process. In the agent interaction model, the coalition formation process will follow a two steps protocol. In the first step, the coalition is formed according to the coalition structure. The second step is used to verify the validation of the coalition and to decide to take the coalition or not.

First step Agent $a_{i}$ sends a coalition proposal to agent $a_{i+1}$, then $a_{i+1}$ responds to this proposal according to Algorithm 2.

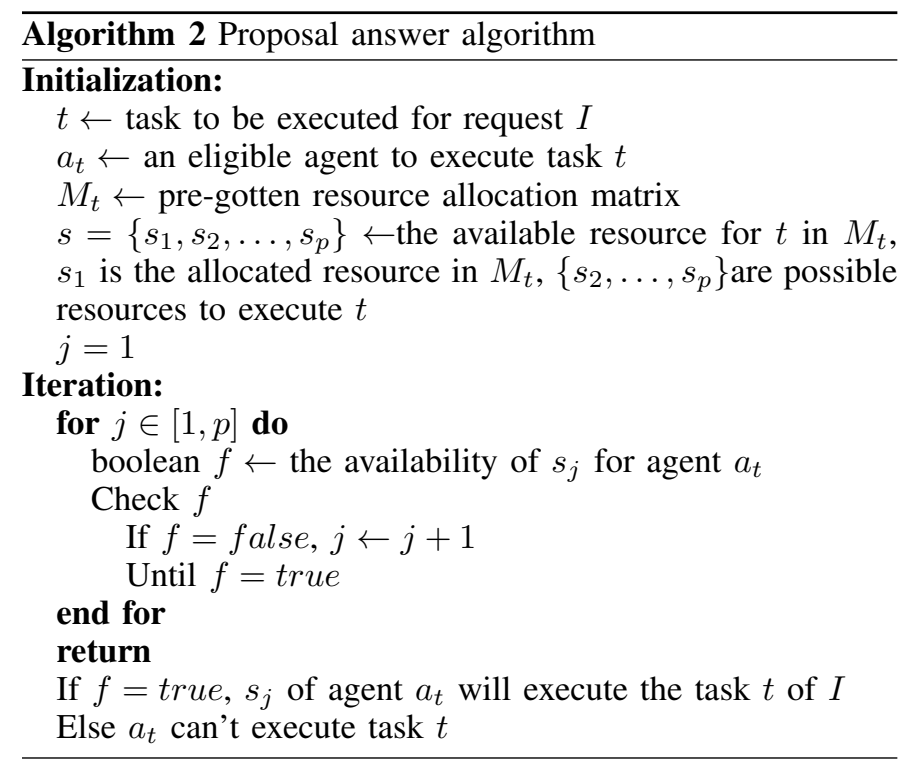

Second step After having obtained the coalition $C_{I}=$ $\left\{a_{I}^{1}\left(s_{1}\right), \ldots, a_{I}^{n}\left(s_{n}\right)\right\}$ for request $I$, the Evaluation agent takes charge of the evaluation of $C_{I}$ with Coalition evaluation algorithm. Then relevant agents update their available resources.

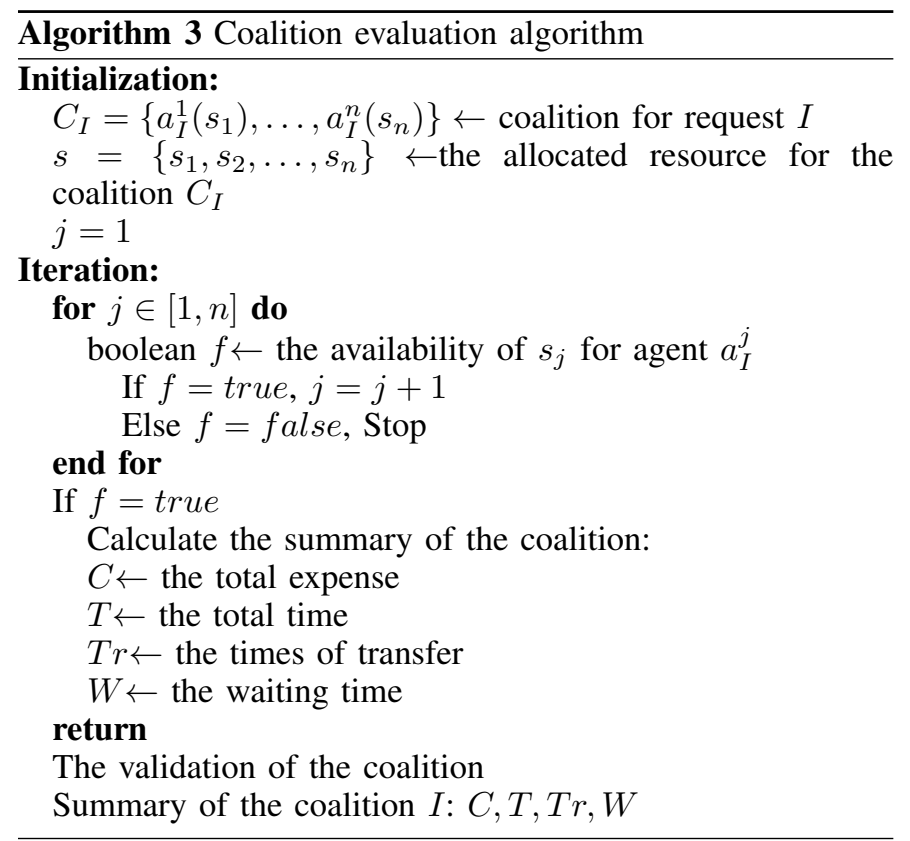

\section{EXEMPLE ILLUSTRATION}

In order to illustrate how the approach proposed in this paper works, an example of simulation will be present as follows.

There are 6 itineraries requests for the correspondent time window and Figure 6 is the map with starting and terminal points, as well as the transfer points.

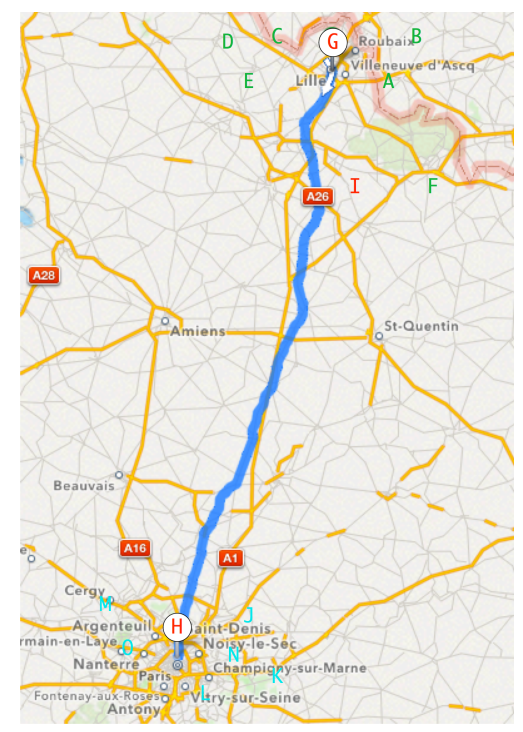

Fig. 6. The requests of itineraries

As showed in Figure 7, we have gotten the shortest itinerary for each request.

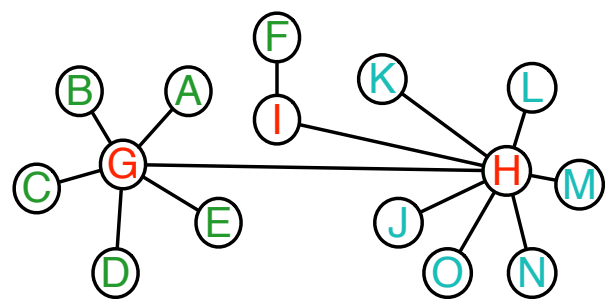

Fig. 7. The graph of the requests

The requests are as follows: $I\left(t_{0}\right)=$ $\left\{I\left(t_{0}\right)_{1}, I\left(t_{0}\right)_{2}, I\left(t_{0}\right)_{3}, I\left(t_{0}\right)_{4}, I\left(t_{0}\right)_{5}, I\left(t_{0}\right)_{6}\right\}$

$I\left(t_{0}\right)_{1}=A \rightarrow O\left[t_{A}, t_{O}\right]$, no carpooling; least expense

$I\left(t_{0}\right)_{2}=B \rightarrow K\left[t_{B}, t_{K}\right]$, no free-use car; least expense

$I\left(t_{0}\right)_{3}=C \rightarrow J\left[t_{C}, t_{J}\right]$, carpooling first; least time

$I\left(t_{0}\right)_{4}=D \rightarrow M\left[t_{D}, t_{M}\right]$, free-use car first; least waiting time

$I\left(t_{0}\right)_{5}=E \rightarrow L\left[t_{E}, t_{L}\right]$, no carpooling; least transfers

$I\left(t_{0}\right)_{6}=F \rightarrow N\left[t_{F}, t_{N}\right]$, no public transport

There are several route segments, $(A, G),(D, G),(E, G)$, $(F, G),(G, H),(H, J),(H, K),(H, L),(H, K)$, etc. which are assured by more than one transportation service. 
The segments $G H$ are shared by several itineraries, thus, the assignment process will be launched to allocate the resource.

$m_{1}$ represents public transport, $m_{2}$ and $m_{3}$ represent carpooling and free-use car, respectively.

Taking account of the users' preferences about the transpiration tools, the itineraries demands become:

$$
\begin{aligned}
I\left(t_{0}\right)_{1} & =\left\{(A, G)_{m_{1}, m_{3}},(G, H)_{m_{1}, m_{3}},(H, O)_{m_{3}}\right\} \\
I\left(t_{0}\right)_{2} & =\left\{(B, G)_{m_{1}},(G, H)_{m_{1}, m_{2}},(H, K)_{m_{1}, m_{2}}\right\} \\
I\left(t_{0}\right)_{3} & =\left\{(C, G)_{m_{1}},(G, H)_{m_{1}, m_{2}, m_{3}},(H, J)_{m_{1}, m_{2}}\right\} \\
I\left(t_{0}\right)_{4} & =\left\{(D, G)_{m_{1}, m_{3}},(G, H)_{m_{1}, m_{2}, m_{3}},(H, K)_{m_{1}, m_{3}}\right\} \\
I\left(t_{0}\right)_{5} & =\left\{(E, G)_{m_{3}},(G, H)_{m_{1}, m_{3}},(H, L)_{m_{1}}\right\} \\
I\left(t_{0}\right)_{6} & =\left\{(F, I)_{m_{2}},(I, H)_{m_{2}},(H, N)_{m_{3}}\right\}
\end{aligned}
$$

With the decomposition of the itineraries, the assignment will occur for each individual segment.

After the assignment, the possible assignment matrix are obtained, for example, the Table II for the segment $(G, H)$. The users' preferences are respected and the constrains like the number of available vehicles are not violated. During the coalition formation, the route agent $a_{R(G, H)}$ carried this matrix.

TABLE II. THE ASSIGNMENT MATRIX FOR SEGMENT $(\mathrm{G}, \mathrm{H})$

\begin{tabular}{|c|c|c|c|}
\hline$R(G, H)$ & Train & Carpooling & Free-use car \\
\hline$P_{1}$ & 1 & 0 & $*$ \\
\hline$P_{2}$ & 1 & $*$ & 0 \\
\hline$P_{3}$ & $*$ & 1 & $*$ \\
\hline$P_{4}$ & $*$ & $*$ & 1 \\
\hline$P_{5}$ & 1 & 0 & $*$ \\
\hline
\end{tabular}

It's possible that it exists more than one feasible solution, for example, $(A, G)$ of the request $I\left(t_{0}\right)_{1}$, both the public transport and the free-use car are possible. In this case, the coalition of the segments in this work will solve this problem by choosing one of these feasible solutions.

For each section of the itineraries, one or several solutions may be proposed. Taking the transit time and several possible departure times (i.e. metro, bus, train) into account, the departure time and the arrival time can also be taken as a condition for the coalition between these segments. The result is ready for the coalition procedure to form the itineraries. After having taken the users' preferences into account for the coalition evaluation agent, the following results are gotten:

$$
\begin{aligned}
& I\left(t_{0}\right)_{1}=\left\{(A, G)_{m_{1}},(G, H)_{m_{1}},(H, O)_{m_{3}}\right\} \\
& A \stackrel{\text { public transport }}{\longrightarrow} G \stackrel{\text { public transport }}{\longrightarrow} H \stackrel{\text { free-use car }}{\longrightarrow} O \\
& I\left(t_{0}\right)_{2}=\left\{(B, G)_{m_{1}},(G, H)_{m_{1}},(H, K)_{m_{1}}\right\} \\
& B \stackrel{\text { public transport }}{\longrightarrow} G \stackrel{\text { train }}{\longrightarrow} H \stackrel{\text { public transport }}{\longrightarrow} K \\
& I\left(t_{0}\right)_{3}=\left\{(C, G)_{m_{1}},(G, H)_{m_{3}},(H, J)_{m_{2}}\right\} \\
& C \stackrel{\text { public transport }}{\longrightarrow} G \stackrel{\text { free-use car }}{\longrightarrow} H \stackrel{\text { carpooling }}{\longrightarrow} J \\
& I\left(t_{0}\right)_{4}=\left\{(D, G)_{m_{3}},(G, H)_{m_{3}},(H, K)_{m_{3}}\right\} \\
& D \stackrel{\text { free-use car }}{\longrightarrow} G \stackrel{\text { free-use car }}{\longrightarrow} H \stackrel{\text { free-use car }}{\longrightarrow} K
\end{aligned}
$$

$$
\begin{aligned}
& I\left(t_{0}\right)_{5}=\left\{(E, G)_{m_{3}},(G, H)_{m_{3}},(H, L)_{m_{3}}\right\} \\
& E \stackrel{\text { free-use car }}{\longrightarrow} G \stackrel{\text { free-use car }}{\longrightarrow} H \stackrel{\text { free-use car }}{\longrightarrow} L \\
& I\left(t_{0}\right)_{6}=\left\{(F, I)_{m_{2}},(I, H)_{m_{2}},(H, N)_{m_{3}}\right\} \\
& F \stackrel{\text { carpooling }}{\longrightarrow} I \stackrel{\text { carpooling }}{\longrightarrow} H \stackrel{\text { free-use car }}{\longrightarrow} N
\end{aligned}
$$

\section{CONCLUSIONS AND FUTURE WORKS}

In this paper, the coalition of the route segments to form an entire itinerary is studied. The combination of different routes represented by the route agents is also studied. The coalition is dedicated to the formation of an optimized itinerary solution for the travelers following their criteria. With the definitions, the agent interaction protocol for the coalition process is established and the agent behaviors are also described.

In the future works, the assignment of the transportation resource for the travelers will joint with this coalition process and an integrated multi-agent system will be detailed for the distributed co-modal transport information system. In addition, the characteristics of different transport modes should be taken into consideration.

\section{REFERENCES}

[1] M. Klusch and O. Shehory, "A polynomial kernel-oriented coalition formation algorithm for rational information agent," in Proc. ICMAS, pp.157-164, 1996

[2] G. Zlotkin and J. Rosenschein, "Coalition, cryptography, and stability: mechanisms for coalition formation in task oriented domains," in Proc. AAAI'94, pp. 432-437, 1994.

[3] T.W. Sandholm, K. Larson etc, "Coalition among computationally bounded agents," Artificial Intelligence, pp.99-137, 1997.

[4] T.W. Sandholm, K. Larson etc, "Coalition structure generation with worst case guarantees," Artificial Intelligence, vol. 111, no. 1-2, pp.209-239, 1999.

[5] O. Shehory and S. Krauss, "Methods for task allocation via agent coalition formation," Artificial Intelliegence, vol. 101, no. 1, pp.165200, 1998

[6] O. Shehory and S. Krauss, "Feasible formation of coalitions among autonomous agents in non-super-additive environments," Computational Intelligence, vol. 15, no. 3, pp. 218-251, 1999.

[7] Z. Wang, K. Mesghouni and S. Hammadi, "A co-modal transport information system in a distributed environment," International Journal of Advanced Computer Science and Information Technology, vol. 3, issue 2, pp. 83-99, 2014.

[8] J. Wang and T. Kaempke, "Shortest route computation in distributed systems," Computers \& Operations Research, vol.31, issue 10, pp.16211633, September 2004.

[9] J. Wang and T. Kaempke, "The fastest itinerary in time-dependent decentralized travel information systems," Journal of Combinatorial Optimization, vol.12, no.3, pp.167-185, 2006.

[10] S. Aknine and O. Shehory, "Coalition Formation: Concessions, Task Relationships and Complexity Reduction," ArXiv Computer Science eprints, Feb. 2005.

[11] I. Müller, R. Kowalczyk and P. Braun, "Toward agent-based coalition formation for service composition," in Intl. Conf. on Intelligent Agent Technology, pp. 73-80, 2006.

[12] T. Génin and S. Aknine, "Coalition formation strategies for self-interested agents in task oriented domains," in Proc. of the IEEE/WIC/ACM Intl. Conf. on Web Intelligence and Intelligence Agent Technology, pp. 205-212, Aug. 2010.

[13] S. Kraus, O. Shehory and G. Taase, "The Advantage of Compromising in Coalition Formation with Incomplete Information," in Proc. of the second Intl. joint Conf. on Autonomous Agents and Multiagent Systems, pp. 588-595, 2004. 